\title{
Photon and Graviton Masses in String Theories
}

\author{
V. Alan Kostelecký(1),(a) and Stuart Samuel ${ }^{(2),(b)}$ \\ ${ }^{(1)}$ Physics Department, Indiana University, Bloomington, Indiana 47405 \\ ${ }^{(2)}$ Physics Department, City College of City University of New York, New York, New York 10031
}

(Received 10 May 1990)

\begin{abstract}
We show that string theories allow interactions potentially leading to photon and graviton mass terms when one or more scalar fields acquire vacuum expectation values. A general analysis is presented for the case when one of these scalars is the electroweak Higgs-boson doublet. The results provide a new constraint on model building for string theories.
\end{abstract}

PACS numbers: 11.17.+y, 04.50.+h, 12.10.Gq, 14.80.Am

Tight experimental upper bounds exist for the photon $^{1-4}$ and graviton ${ }^{5}$ masses. In standard theories of electrodynamics and general relativity, whether unified or not, these particles are exactly massless because unbroken gauge invariances preclude mass terms.

String theory, which is based on an extended object, provides a promising and different approach to unification. Particles correspond to different vibrational modes. Interactions are spread over a Planck length, softening forces at short distances and leading to an ultravioletfinite theory. In this Letter, we demonstrate that a class of stringy interactions can lead to mass terms for gauge bosons, in particular, for the photon and graviton. The appearance of these new terms is a consequence of the extended nature of strings.

The mass terms of interest arise via collective effects in string interactions. These effects can be examined directly in a given string model via covariant field theory. Such field theories are now available for the open bosonic string, ${ }^{6}$ the closed bosonic string, ${ }^{7}$ and the open superstring. ${ }^{8}$ These models may therefore be studied to seek insights into the nature of multistring effects.

When each string mode is described by an ordinary particle field, the action contains an infinite number of kinetic and interaction terms. One striking feature is the appearance of interaction terms of the form $S^{n} T \cdot T$, where $S$ is a Lorentz-scalar field and $T$ is a Lorentz tensor. Such interactions are truly stringy: Gauge invariances and renormalizability generically exclude them from the usual particle field theories of gauge and/or gravitational interactions. ${ }^{9}$ In string theories, the gauge invariance is maintained by cancellations among the expressions arising from a gauge transformation.

Scalar-tensor-tensor terms of this type do occur in some string theories. A simple example is provided by the tachyon-vector-vector coupling in the open bosonic string. The Lagrangian contains the terms

$$
\operatorname{tr}\left(-\frac{1}{2 \alpha^{\prime}} \phi^{2}-\frac{3 \sqrt{3}}{8} g \phi A_{\mu} A^{\mu}+\cdots\right),
$$

where $\phi$ is the tachyon, $A_{\mu}$ is the vector, and $g$ is the on- shell three-tachyon coupling. Naively, the second term is not invariant under the gauge transformation $\delta A_{\mu}$ $=\partial_{\mu} \lambda$. However, at order $g$ the tachyon gauge transformation includes the term $\delta \phi=-(3 \sqrt{3} / 4) g \alpha^{\prime} A^{\mu} \partial_{\mu} \lambda$. The latter makes the tachyon mass term also naively noninvariant. However, the two extra pieces cancel when combined.

The new mass terms arise from the stringy $S^{n} T \cdot T$ interactions ${ }^{10}$ if one or more scalars $S$ acquires a vacuum expectation value (VEV). For the open bosonic string, it has been shown ${ }^{11}$ that nonperturbative, nonzero scalar VEVs give rise to a mass term for $A_{\mu}$. In general, the consequences for physics depend on the nature and size of the scalar VEVs.

Let us define a strongly nonperturbative string vacu$\mathrm{um}^{12}$ as one in which one or more scalar fields have VEVs of order of the Planck mass $M_{P}$. Such vacua are natural to strings since the only fundamental scale is the Regge slope $\alpha^{\prime} \approx 1 / M_{P}^{2}$. However, there are reasons for believing a realistic string theory might not be strongly nonperturbative. In the canonical perturbative vacuum, superstrings generate a wide range of features seen in nature: a massless graviton, massless gauge bosons coupling correctly to chiral fermions, and gauge groups large enough to incorporate $\mathrm{SU}_{c}(3) \otimes \mathrm{SU}_{L}(2) \otimes \mathrm{U}_{Y}(1)$. The ingredients are in place for a complete unification of the four fundamental forces. In contrast, investigations ${ }^{11}$ for bosonic strings indicate that the physics in a strongly nonperturbative vacuum is unlikely to resemble that of the canonical vacuum. Since the physics is approximately correct in the latter, it seems unnatural to search for a radically different ground state.

Nonetheless, nature cannot be in the canonical perturbative vacuum. The $\mathrm{SU}_{L}(2)$-doublet Higgs boson $H$ of the electroweak model, which is perturbatively a tachyon, acquires a VEV $\langle H\rangle \ll M_{P}$. We call weakly nonperturbative any string vacuum in which all scalar VEVs are small compared to $M_{P}$. It seems that a realistic string model has a weakly nonperturbative ground state.

In this Letter, we investigate the possibility that photon and graviton mass terms arise in string theories from scalar VEVs smaller than $M_{P}$. The issue has import be- 
cause there is at least one scalar field in nature, $H$, with a nonzero VEV. We show that, although $\langle H\rangle$ is miniscule compared to $M_{P}$, sizable photon and graviton mass terms can be generated.

A source of uncertainty is the identification of $H$ with a field in string theory: $H$ could be a fundamental scalar, it could be composite, or it could arise during compactification. In certain models, there are other scalars with nonzero VEVs. Our main point is that for every scalar operator in a string model one must verify that the photon and graviton do not acquire masses above the current stringent experimental limits.

Let us perform a model-independent analysis when there is a scalar field $S$ with $\langle S\rangle \approx R M_{P}$, where $R \ll 1$. One choice for $S$ is the electroweak $\mathrm{SU}_{L}(2)$-doublet $H$, which has $\langle H\rangle \approx 175 \mathrm{GeV}$, i.e., $R \approx 10^{-17}$. However, other possibilities exist. First, in some generalizations of the standard model, $\mathrm{SU}_{c}(3) \otimes \mathrm{SU}_{L}(2) \otimes \mathrm{U}_{Y}(1)$ singlets acquire VEVs. Second, in compactified string theories $S$ might be a higher-dimensional tensor but a $D=4$ scalar. For example, in Wilson-line breaking ${ }^{13,14} S$ is a higher component of a gauge boson. In contrast, $S$ is probably not the dilaton, which is believed to couple only through derivative interactions.

In the four-dimensional effective theory, the interactions of interest have the form

$$
I_{n} \propto q^{n} \alpha^{\prime(n-2) / 2} \int d^{4} x S^{n} A_{T} A^{T},
$$

where $A_{T}$ is the photon $A_{\mu}$ or the graviton $h_{\mu v}$, and $q$ is the gauge coupling. For the open strings $q^{2}$ $\approx V^{-1 / 2} \sqrt{8 \pi G} \alpha^{\prime(D-6) / 4}$, where we take the compactification volume $V \approx M_{P}^{4 \mathrm{D}}$, and only $n=1$ terms occur explicitly in the field theory. However, terms with $n \geq 2$ can be generated by integrating over heavy fields. For the heterotic strings, $\alpha^{\prime} q^{2} \approx 16 \pi G$. In any case, the interactions $I_{n}$ produce mass terms $m_{A_{T}} \approx R^{n / 2} M_{P}$, which are very small for large $n$.

Experimental data place constraints on $n$. The current limit on the photon mass is ${ }^{1} m_{\gamma}<6 \times 10^{-25} \mathrm{GeV}$. It is a secure bound based on observation of Jupiter's magnetosphere during the Pioneer-10 mission. A bound as strict as $m_{\gamma}<3 \times 10^{-36} \mathrm{GeV}$ has been conjectured ${ }^{2}$ from known and speculated properties of galactic magnetic fields. Other estimates ${ }^{3,4}$ range from $3 \times 10^{-28}$ to 3 $\times 10^{-32} \mathrm{GeV}$. For the graviton, one has $m_{h}<10^{-38}$ $\mathrm{GeV}$ from observations of gravitationally bound clusters of galaxies. ${ }^{5}$

For $R \approx 10^{-17}$, these bounds show the necessity of excluding interactions $I_{n}$ for $n \lesssim 7$. Note that the larger the VEV $\langle S\rangle$, the more powers of $n$ must be considered, and therefore the greater the constraints on $n$. For example, grand-unified theories and typical string models undergo symmetry breakdown at an intermediate mass scale $M_{I} \approx 10^{15} \mathrm{GeV}$. This involves a scalar $S$ with $\langle S\rangle \approx R M_{P}, R \gtrsim 10^{-4}$, which requires the absence of terms $S^{n} A_{T} A^{T}$ for $n \lesssim 35$. Arguments must be found to exclude such interactions if these theories are to be experimentally viable.

The results apply if the electroweak model is broken by a technicolor ${ }^{15}$ condensate $\langle\bar{\psi} \psi\rangle$, although fewer dangerous terms occur. Possible masses arise from interactions such as

$$
q^{n} \alpha^{(3 p-2) / 2} \int d^{4} x(\bar{\psi} \psi)^{p} A_{T} A^{T}
$$

Since $\langle\bar{\psi} \psi\rangle \approx(600 \mathrm{GeV})^{3}, m_{A_{T}} \approx R^{3 p / 2} M_{P}$ with $R$ $\approx 2 \times 10^{-16}$. Only $p \leq 2$ conflicts with experiment.

In certain cases, some interactions can automatically be absent, perhaps due to discrete or continuous symmetries or supersymmetries. For example, for the $\mathrm{SU}_{L}$ (2)-doublet $H$ the odd- $n$ terms $H^{n} A_{T} A^{T}$ cannot appear for group-theoretical reasons. Another escape is seen in the open string with Paton-Chan factors if the photon generator and the Wilson-line expectation $\left\langle A_{I}\right\rangle$ $=\langle S\rangle$ are orthogonal in group space. Since open-string amplitudes involve a single trace, no photon mass term is produced. Even when generators overlap, the trace may vanish because commutators appear. However, since closed strings and gravity appear at loop level in open strings, graviton mass terms must still be checked.

Let us next consider the situation for individual string theories. The field theory of the open bosonic string does have interactions $I_{n} \sim S^{n} T \cdot T$. An explicit example with $n=1$ is provided by the tachyon-vector-vector coupling discussed above. In a stable vacuum, the tachyon must have a nonzero expectation value and a photon mass term is generated. ${ }^{16}$

Next, consider the closed bosonic string. Among the cubic interactions in the nonpolynomial string field theory one discovers the term $\left(3^{3} / 2^{5}\right) g \varphi h_{\mu \nu} h^{\mu \nu}$, where $\varphi$ is the closed-string tachyon, $h_{\mu \nu}$ is the graviton, and $g$ is the on-shell coupling between three closed-string tachyons. This term is of the form $I_{1}$. When $\langle\varphi\rangle \neq 0$ in the true vacuum, a mass term is produced for the graviton. ${ }^{17}$

A covariant string field theory is also known for the open superstring. Since there is no tachyon, the lowenergy limit can be taken directly. To lowest order, this limit yields an ordinary supersymmetric gauge field theory. One therefore expects no terms $I_{n}$ for $n \leq 2$. However, there is no evident reason why terms $n \geq 3$ should be absent in general. Such terms would appear as nonrenormalizable corrections to the low-energy theory. Their apparent lack of gauge invariance is misleading: They combine with other nonrenormalizable corrections to maintain gauge invariance. Any effort to construct a realistic model involving nonzero scalar expectations needs to provide a mechanism whereby gauge mass terms are absent or suppressed.

Heterotic strings ${ }^{18-20}$ are promising candidates for a realistic phenomenology. ${ }^{14,21}$ There are two types of $D=10$ heterotic strings: those without tachyons ${ }^{18,19}$ and those with tachyons. ${ }^{20}$ The latter theories are still of phenomenological interest because the tachyon $\phi$ might 
yield spontaneous Lorentz-symmetry breaking ${ }^{22}$ or grand-unified symmetry breaking.

A direct analysis is hampered by the absence of a heterotic-string field theory. For the heterotic strings with tachyons, one can use conformal field theory methods to investigate amplitudes that would arise from interactions $I_{1}$ in toroidally compactified strings. Here, we consider $\phi A_{T} A^{T}$ interactions.

To perform the off-shell calculation, ${ }^{23,24}$ one needs vertex operators for the states. They are the product of a left vertex operator $V_{L}$ with a right vertex operator $V_{R}$. For $A_{T}, V_{L}^{A_{\mu \nu}}(\bar{z})=\partial X^{v} \exp (k \cdot X)(\bar{z})$, and $V_{L}^{A_{\mu}}(\bar{z})=\partial X^{I}$ $\times \exp (k \cdot X)(\bar{z})$ or $\exp \left(\sum_{I} k^{I} X^{I}\right) \exp (k \cdot X)(\bar{z})$. Here, the $X^{I}$ are compactified bosons, $k^{I} \in \Lambda$, where $\Lambda$ is a lattice, and $\sum_{I} k^{I} k^{I}=2$. Equations (4.1b) and (4.1f) of Ref. 24 provide $V_{R}^{A} T(z)$. For the tachyon, $V_{L}^{\phi}=\exp \left(\sum_{I} k^{I} X^{I}\right)$ $\times \exp (k \cdot X)(\bar{z})$ with $\sum_{I} k^{I} k^{I}=1$, while $V_{R}^{\phi}=c \exp (-\varphi)$ $\times \exp (k \cdot X)(z)$ and $V_{R}^{\phi}=(c k \cdot \psi-\gamma / 2) \exp (k \cdot X)(z)$ in the $(-1)$ and $(0)$ pictures, respectively. Here, $\varphi$ is the first-quantized spinor ghost.

We find ${ }^{25}$

$I_{1} \propto\left\langle V_{L}^{\phi}\left(\bar{z}_{1}\right) V_{R}^{\phi}\left(z_{1}\right) V_{L}^{A_{T}}\left(\bar{z}_{2}\right) V_{R}^{A_{T}}\left(z_{2}\right) V_{L}^{A_{T}}\left(\bar{z}_{3}\right) V_{R}^{A_{T}}\left(z_{3}\right) O\right\rangle$,

where $O=X(i)$ in the $(-1)$-picture formalism ${ }^{26}$ and $O=Y_{-2}$ in the (0)-picture formalism. ${ }^{8}$ Here, $X(z)$ is the picture-changing operator ${ }^{27}$ and $Y_{-2}=Y(-i) Y(i)$ is a $(-2)$ inverse picture-changing operator, ${ }^{8}$ where $Y(z)=c \partial \xi \exp (-2 \varphi)$.

The correlations factorize into a product of left and right sectors. Explicit calculation reveals the right-sector factor is zero due to conservation of ghost and $\psi$-fermion numbers. The left-sector expectation is also zero: There is no group invariant involving one vector representation with two adjoint representations. Thus, $\phi A_{T} A^{T}$ terms are absent.

There are three heterotic strings without tachyons: ${ }^{18-20} E_{8} \otimes E_{8}, S O(32)$, and $S O(16) \otimes S O(16)$. Using the fact that the low-energy limit is an ordinary supergravity theory, one expects there are no terms of the form $I_{n}$ with $n \leq 2$. To verify this, consider $A_{I}$ as a candidate for $S$, where $A_{I}$ is a component of $A_{\mu}$ with $\mu \geq 4$ in a theory with Wilson-line breaking for which $\left\langle A_{I}\right\rangle \neq 0$.

Explicit calculation shows that there is no static $n=1$ term. For $n=2$, consider the four-vector-boson amplitude given in Eq. (5.17) of Ref. 19. Graphs with massless particles appearing in propagators must be subtracted. The terms proportional to a single trace of four matrix generators are not problematic because commutators appear. The heterotic string reproduces the quartic vector-boson coupling $\operatorname{tr}\left(\left[A_{\mu}, A_{v}\right]\left[A^{\mu}, A^{v}\right]\right)$ in the low-energy limit, as one can check. Thus, the dangerous terms are of the form $\operatorname{tr}() \operatorname{tr}()$. However, these vanish in the static limit $s=t=u=0$.

The two-graviton two-vector-boson amplitude can also be obtained using the results of Ref. 19. All terms vanish in the static limit, so Wilson-line breaking in the quartic interactions does not lead to graviton mass terms either.

Although no terms with $n \leq 2$ appear in these strings, there is no apparent reason to exclude the nonrenormalizable corrections $I_{n}$ with $n \geq 3$. Identification in the first-quantized formulation of nonvanishing amplitudes coming from such terms could, in principle, provide direct evidence for their existence.

In summary, we have shown that stringy interactions can allow scalar VEVs to generate photon and graviton mass terms. In particular, one scalar, the Higgs field, is known to have a nonzero VEV. For each candidate string theory, it must be explicitly argued that photon and graviton mass terms above experimental bounds are absent.

Under certain circumstances, string theories might produce photon and graviton masses just below the current experimental limits. For example, the term $I_{7}$ with $R \approx 10^{-17}$ produces a borderline effect that might be revealed by improved experiments. A measurement with a positive result could thereby provide experimental support for strings.

We thank Michael Martin Nieto for discussions on the experimental constraints for photon and graviton masses, and Mike Duff for stimulating correspondence. This work is supported in part by the U.S. Department of Energy under Contracts No. DE-AC02-84ER40125 and No. DE-AC02-83ER40107.

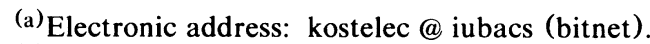

(b) Electronic address: samuel @ ccnysci (bitnet).

${ }^{1}$ L. Davis, Jr., A. S. Goldhaber, and M. M. Nieto, Phys. Rev. Lett. 35, 1402 (1975).

2J. C. Byrne, Astrophys. Space Sci. 46, 115 (1977).

${ }^{3}$ E. Williams and D. Park, Phys. Rev. Lett. 26, 1651 (1971).

${ }^{4}$ A. S. Goldhaber and M. M. Nieto, Rev. Mod. Phys. 43, 277 (1971).

${ }^{5}$ A. S. Goldhaber and M. M. Nieto, Phys. Rev. D 9, 1119 (1974).

${ }^{6}$ E. Witten, Nucl. Phys. B268, 253 (1986).

${ }^{7}$ T. Kugo and K. Suehiro, Kyoto University Report No. KUNS 988 (to be published); T. Kugo, H. Kunimoto, and K. Suehiro, Phys. Lett. B 226, 48 (1989); M. Saadi and B. Zweibach, Ann. Phys. (N.Y.) 192, 213 (1989); M. Kaku, Phys. Rev. D 41, 3734 (1990).

${ }^{8}$ C. Preitschopf, C. B. Thorn, and S. Yost, Nucl. Phys. B337, 639 (1990); I. Ya. Aref'eva, P. B. Medvedev, and A. V. Zubarev, V. A. Steklov Mathematic Institute Report No. SMI10-1989 (to be published).

${ }^{9}$ For the special case $n=2$, terms of this form can arise in particle field theories in the context of the Higgs mechanism.

${ }^{10}$ Note that the mass terms do not arise from the standard Higgs mechanism, with the possible exception of the case $n=2$. Also, in contrast to the Higgs case, the stringy mechanism does not necessarily conserve the total number of light degrees of freedom. This is possible despite the manifest unitarity of the theory because of the presence of an infinite number of states 
and interactions and because of the stringy modifications to the usual particle gauge transformations.

${ }^{11}$ V. A. Kostelecký and S. Samuel, Phys. Rev. Lett. 64, 2238 (1990); Nucl. Phys. B336, 263 (1990); Phys. Rev. D 42, 1289 (1990).

${ }^{12}$ The terminology is used because, at the semiclassical level, any nonzero VEVs are necessarily nonperturbative in the string coupling $g$. See V. A. Kostelecký and S. Samuel, Phys. Lett. B 207, 169 (1988).

${ }^{13}$ Y. Hosotani, Phys. Lett. 126B, 303 (1983).

${ }^{14} \mathrm{P}$. Candelas, G. Horowitz, A. Strominger, and E. Witten, Nucl. Phys. B258, 46 (1985).

${ }^{15}$ S. Weinberg, Phys. Rev. D 13, 974 (1976); 19, 1277 (1979); L. Susskind, Phys. Rev. D 20, 2619 (1979).

${ }^{16}$ No general principle such as gauge invariance can guarantee the masslessness of a gauge boson, or the Higgs effect could not occur. The reader is invited to verify that the existence of the term $\operatorname{tr}\left(\phi A_{\mu} A^{\mu}\right)$ is consistent with the on-shell first-quantized theory by evaluating the expectation $\left\langle c \exp \left(k_{1} \cdot X\right)\left(z_{1}\right) c \partial X_{\mu} \exp \left(k_{2} \cdot X\right)\left(z_{2}\right) c \partial X^{\mu} \exp \left(k_{3} \cdot X\right)\left(z_{3}\right)\right\rangle \neq 0$. This shows that the analysis is independent of the off-shell extension.

${ }^{17}$ The graviton terms are unlikely to represent a cosmological constant because the same mechanism generates a photon mass in the open bosonic string. In any case, the observable consequences of such a cosmological term would make the bounds in this paper even more stringent.

${ }^{18}$ D. J. Gross, J. A. Harvey, E. Martinec, and R. Rohm, Phys. Rev. Lett. 54, 502 (1985); Nucl. Phys. B256, 253 (1985).

${ }^{19}$ D. J. Gross, J. A. Harvey, E. Martinec, and R. Rohm, Nucl. Phys. B267, 75 (1986).

${ }^{20}$ N. Seiberg and E. Witten, Nucl. Phys. B276, 272 (1986); L. Alvarez-Gaumé, P. Ginsparg, G. Moore, and C. Vafa, Phys. Lett. B 171, 155 (1985); L. J. Dixon and J. A. Harvey, Nucl. Phys. B274, 93 (1986).

${ }^{21}$ R. Arnowitt and P. Nath, Phys. Rev. Lett. 62, 2225 (1989); Phys. Rev. D 40, 191 (1989), and references therein.

${ }^{22}$ V. A. Kostelecký and S. Samuel, Phys. Rev. D 39, 683 (1989); Phys. Rev. Lett. 63, 224 (1989); Phys. Rev. D 40, 1886 (1989).

${ }^{23}$ S. Samuel, Nucl. Phys. B308, 317 (1988); O. Lechtenfeld and S. Samuel, Nucl. Phys. B310, 254 (1988).

${ }^{24} \mathrm{We}$ follow the notation in Refs. 11 and 23 and in V. A. Kostelecký, O. Lechtenfeld, W. Lerche, S. Samuel, and S. Watamura, Nucl. Phys. B288, 173 (1987).

${ }^{25}$ The proportionality factor involves momentum-dependent conformal factors, a coupling constant, and a combinatorial coefficient.

${ }^{26}$ O. Lechtenfeld and S. Samuel, Phys. Lett. B 213, 431 (1988).

${ }^{27}$ D. Friedan, E. Martinec, and S. Shenker, Nucl. Phys. B271, 93 (1986). 